\title{
MRI in Acute Post-Traumatic Spinal Cord Injury: Assessment of MRI Findings in Predicting Prognosis
}

\author{
Naveen K G ${ }^{1}$, T Arul Dasan², Rupesh $\mathbf{G}^{3}$, Neeraja Akki ${ }^{4}$ \\ ${ }^{1}$ Assistant Professor, Department of Radiodiagnosis, Bangalore Medical College and Research Institute, Bangalore, \\ Karnataka - 560002, ${ }^{2}$ Professor and Head, Department of Radiodiagnosis, Bangalore Medical College and Research Institute, \\ Bangalore, Karnataka - 560002, ${ }^{3}$ Postgraduate, Department of Radiodiagnosis, Bangalore Medical College and Research \\ Institute, Bangalore, Karnataka - 560002, ${ }^{4}$ Postgraduate, Department of Radiodiagnosis, Bangalore Medical College and \\ Research Institute, Bangalore, Karnataka - 560002, India
}

Corresponding author: Dr. Neeraja Akki, Plot No 92, Aditya Hospital, Bhairav Nagar, Swatantra Yodhar Colony, Vijayapur, Karnataka - 586102, India

DOI: http://dx.doi.org/10.21276/ijcmsr.2020.5.1.57

How to cite this article: Naveen K G, T Arul Dasan, Rupesh G, Neeraja Akki. MRI in acute post-traumatic spinal cord injury: assessment of MRI findings in predicting prognosis. International Journal of Contemporary Medicine Surgery and Radiology. 2020;5(1):A258-A262.

\section{A B S T R A C T}

Introduction: Magnetic Resonance Imaging plays an essential role in evaluation of acute spinal trauma. It provides adequate information about neural and extra neural injuries requiring surgical interventions. In cases of spinal cord oedema, contusion, haemorrhage and ischemia, MRI findings may serve as prognostic indicators. The aim of this study was to provide a general overview of the standards for initial assessment and to predict the outcome measurement.

Material and methods: A prospective study from November 2016 to May 2018 was carried out on 100 patients presenting with acute spinal trauma. MR imaging of spine was done for cases clinically suspected to have acute spinal cord injury. Detailed neurological examination of the patient was done on admission and the time of discharge.

Results: Cord haemorrhage and oedema were the two most common findings on MR Imaging of the injured spinal cord, seen in $25 \%$ and $32 \%$ of patients respectively. Majority of the MRI findings correlated well with the clinical profile of the patient.

Conclusions: MR Imaging is the most sensitive modality for prospective detection of spine cord injuries, the cord findings correlate with the neurological deficit of the patient on admission and discharge. MRI is useful for initial diagnosis of acute spinal cord injury and its prognostication for predicting neurological recovery.

Keywords: Acute Spinal Cord Injury, MRI, Prognostication, Spinal Trauma

\section{INTRODUCTION}

The Acute traumatic spinal cord injury (SCI) is any form of insult to the spinal cord, either permanent or temporary, which will result in loss of its sensory, motor or autonomic function. Though SCI can result from non-traumatic or traumatic aetiologies, this study will deal with traumatic SCI. Conventional Radiography and Computed Tomography are first line investigations during emergencies, but the advent of MR imaging has made it possible to accurately characterize the underlying spinal cord injury even when conventional studies are normal. Subtle bone marrow, soft-tissue, and spinal cord abnormalities can be readily detected on MRI. The aim of this study was to describe the MR features in acute spinal cord injury and correlate MR findings with the neurological deficits of patients on admission and to assess role of MR findings in predicting prognosis within 15 days following trauma.

\section{MATERIAL AND METHODS}

A hospital based prospective study was conducted on 100 patients with history of trauma, clinically suspected of having spinal cord injury, presenting to Victoria Hospital and Bowring \& Lady Curzon Hospital attached to Bangalore Medical College and Research Institute, Bengaluru, from November 2016 to May 2018. Patients were subjected to MRI examination of spine. Detailed neurological examination of each patient was done prior to the scan and repeated at the time of discharge.

All MR imaging were performed on a 1.5-T Siemens Avanto Magnetom MR system. The study was performed with patient in supine position with quiet breathing obtaining sagittal T2 and T1-weighted fast spin echo images, STIR, GRE, coronal STIR and axial T2 and T1-weighted fast spin echo images, GRE. Sagittal DWI in patients with edematous T2 hyperintense cord and in patients with abnormal clinical findings and normal conventional MR sequences.

\section{Inclusion criteria}

All patients suspected of having acute spinal cord injury referred to the department of Radiodiagnosis were included in the study after taking written informed consent. 


\section{Exclusion Criteria}

1. Patients with severe coexistent other systemic injuries; Concomitant head Injury and Glasgow coma scale score $<15$.

2. Contraindications to MRI such as pacemakers, aneurysmal clips, cochlear implants etc.

3. Patients with pre-existing spinal cord pathology

4. Claustrophobic patients.

MR images were analysed and correlated with findings on neurological examination according to American Spinal Injury Association (ASIA) impairment scale (AIS) at the time of MRI examination and subsequently at subacute interval to assess neurological outcome.

Resuscitation apparatus and emergency drugs were kept ready.

MRI findings were recorded. The following findings were identified and considered for the study:

1. Cord haemorrhage.

2. Cord oedema

3. Cord ischemia.

4. Cord transection.

5. Normal cord.

\section{Clinical assessment of spinal cord injury}

A standardized physical examination as endorsed by the International Standards for Neurological and Functional Classification of Spinal Cord Injury Patients, also commonly called the American Spinal Injury Association (ASIA) guidelines was performed. A detailed motor and sensory examination of the patient was done and graded according to American Spinal Injury Association Scale which is as follows

A Complete: No motor or sensory function is preserved in the sacral segments S4-S5.

B Incomplete: Sensory but not motor function preserved below the neurologic level and includes the sacral segments $\mathrm{S} 4-\mathrm{S} 5$.

C Motor function is preserved below the neurologic level, and more than half of the key muscles below the neurologic level have a muscle grade less than 3 .

D Incomplete: Motor function is preserved below the neurologic level, and at least half of key muscles below the neurologic level have a muscle grade of 3 or more.

E Normal: Motor and sensory function are normal.

\section{STATISTICAL ANALYSIS}

The strength of association between extent of spinal cord injury and outcome was described using Odds ratio and correlation co efficient. Chi square test of significance $(p<0.005)$ was used to assess the association between MR findings and clinical outcome.

\section{RESULTS}

Data of all 100 cases of acute spinal cord injury was evaluated. The following observations were made.

\section{Demographic data}

Mean age was 36.24 and the age ranged from 14-72 years (table-). Our study had a male predominance, with 72 male patients and 28 female patients.

\begin{tabular}{|l|c|c|}
\hline Age group in years & No. of cases & Percentage \\
\hline 11 to 20 & 9 & 9 \\
\hline 21 to 30 & 23 & 23 \\
\hline 31 to 40 & 26 & 26 \\
\hline 41 to 50 & 19 & 19 \\
\hline 51 to 60 & 13 & 13 \\
\hline 61 to 70 & 6 & 6 \\
\hline 71 to 80 & 4 & 4 \\
\hline Total & 100 & 100 \\
\hline \multicolumn{2}{|c|}{ Table-1: Age wise distribution of cases } \\
\hline
\end{tabular}

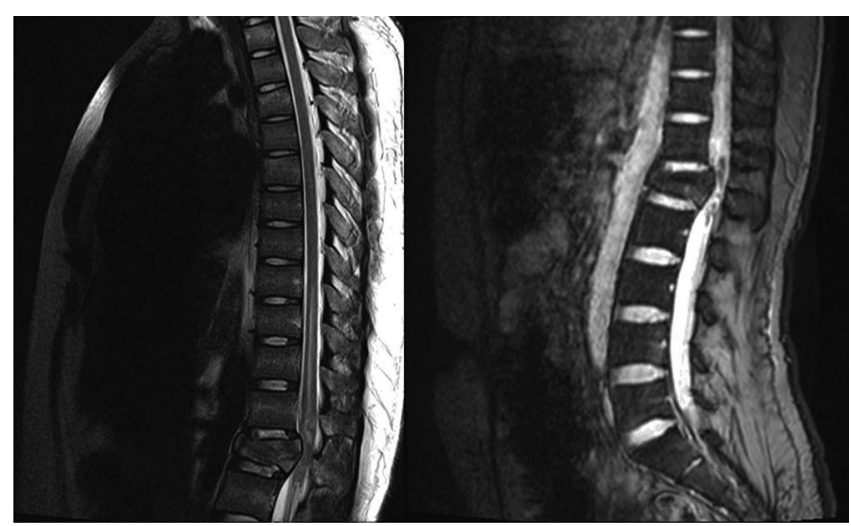

Figure-1 a, b: A 20 year old male with history of fall from height presented with inability to move lower limbs. T2 sagittal (a) image shows burst fracture of L1 with spinal canal narrowing causing cord compression and hyper intensity in the cord from the level of D9 to conus, suggestive of cord oedema. Sagittal GRE (b) shows loss of signal within the cord at the level of oedema, suggestive of cord haemorrhage.

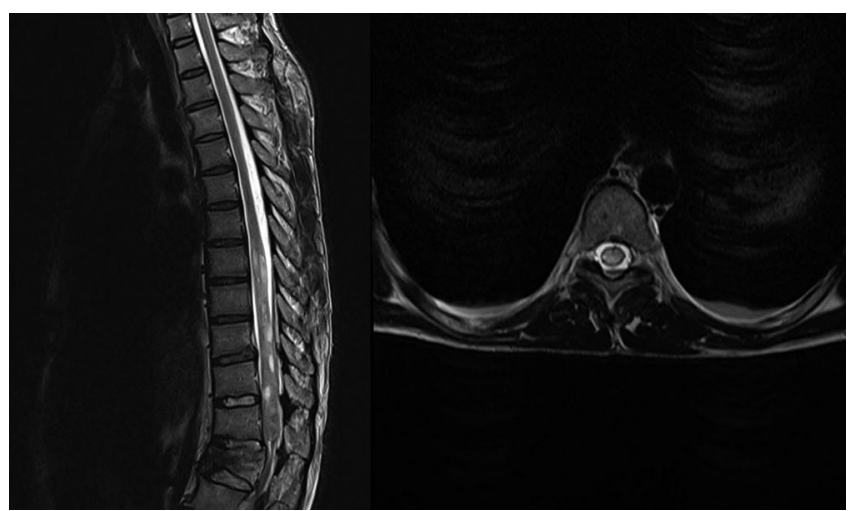

Figure-2 a, b: A 26 year old male with history of road traffic accident presented with inability to move lower limbs. T2 sagittal (a) image shows anterior wedge compression of L1 vertebra with spinal canal narrowing and ill-defined hyper intensity in the cord from D8 to L1 level, suggestive of cord oedema.T2 axial (b) image shows a fluid level in the posterior thecal sac suggestive of epidural haemorrhage

\section{Spinal cord changes on MRI}

Among 100 cases, 33\% of cases showed cord oedema (15\% cases with oedema extending $<3 \mathrm{~cm}$ of the cord length and $18 \%$ cases with oedema $>3 \mathrm{~cm}$ of cord length), which was the most common pattern. $28 \%$ of cases had haemorrhagic contusion. $8 \%$ of cases had cord transection. $4 \%$ cases had 


\begin{tabular}{|l|l|c|c|c|c|c|c|}
\hline AIS & & A & B & C & D & E & Total \\
\hline Cord haemorrhage & No. of patients at admission & 16 & 11 & 1 & 0 & 0 & 28 \\
\hline & No. of patients at discharge & 13 & 11 & 4 & 0 & 0 & 28 \\
\hline Cord oedema less than 3 cm & No. of patients at admission & 1 & 2 & 1 & 11 & 0 & 15 \\
\hline & No. of patients at discharge & 1 & 2 & 0 & 10 & 2 & 15 \\
\hline Cord oedema more than 3 cm & No. of patients at admission & 13 & 1 & 2 & 2 & 0 & 18 \\
\hline & No. of patients at discharge & 11 & 0 & 2 & 5 & 0 & 18 \\
\hline Cord transection & No. of patients at admission & 8 & 0 & 0 & 0 & 0 & 8 \\
\hline & No. of patients at discharge & 8 & 0 & 0 & 0 & 0 & 8 \\
\hline Cord ischemia & No. of patients at admission & 3 & 1 & 0 & 0 & 0 & 4 \\
\hline & No. of patients at discharge & 3 & 1 & 0 & 0 & 0 & 4 \\
\hline No cord findings & No. of patients at admission & 0 & 1 & 2 & 2 & 22 & 27 \\
\hline \multicolumn{2}{|l|}{ No. of patients at discharge } & 0 & 1 & 0 & 0 & 26 & 27 \\
\hline
\end{tabular}

\begin{tabular}{|l|c|c|}
\hline Outcome & Odds ratio (95\% & $\begin{array}{c}\mathbf{P} \\
\text { value }\end{array}$ \\
\hline 0 Improved, I Not improved & confidence limit) & \\
\hline Cord haemorrhage & $6.73(1.17-38.63)$ & 0.032 \\
\hline 0 absent, I present & & \\
\hline Cord oedema less than 3 cms. & $0.35(0.44-1.87)$ & 0.149 \\
\hline 0 no oedema, I present & $0.41(0.59-31.5)$ & 0.178 \\
\hline Cord oedema more than 3 cms. & & \\
\hline 0 no oedema, I present & Table-3: Various cord findings and their effect on outcome \\
(Multivariate Analysis) & \\
\hline \multicolumn{2}{|c|}{}
\end{tabular}

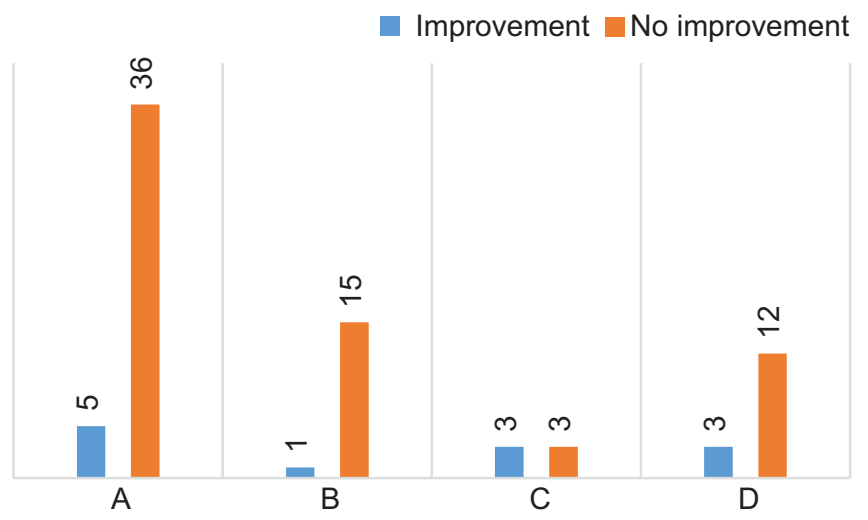

Chart-1: Outcome in patients of spinal trauma according to ASIA impairment Scale

cord ischemia. No cord changes were seen in $27 \%$ of patients, suggesting a clinical diagnosis of Cord concussion.

In 27 patients with no cord findings 22 patients had no clinical deficit and hence graded as AIS E (81.48\%). 1(3.7\%) patient was initially graded as AIS B. 2 patients (7.4\%) were initially graded AIS C and 2 patients were graded AIS D. The patient with AIS B showed no improvement. Both the patients of AIS C and AIS D showed improvement to AIS $\mathrm{E}$ (table-2).

Out of 28 cases of cord haemorrhage, 3 cases showed clinical improvement (11\%). Out of 15 cases with cord oedema < $3 \mathrm{~cm}, 2$ cases showed clinical improvement (13\%). Out of 18 cases with cord oedema $>3 \mathrm{~cm}, 3$ cases showed clinical improvement (17\%). None of the cases with cord ischemia ( 4 cases) and cord transection (8 cases) showed clinical improvement (table-3).

Out of 27 cases with no cord findings, 4 cases showed clinical improvement (15\%) (fig-1).

\section{DISCUSSION}

The results of the study have shown that imaging findings correlate with the clinical neurological examination findings according to the ASIA impairment scale.

Out of 100 patients, 72 were male and 28 were females. The age group commonly affected were those in the age group of 21 - 40 years $(49.0 \%)$. This was in concordance with Shih $\mathrm{P}$. et $\mathrm{al}^{1}$ which showed that the spinal trauma was more common in men and the age group of 25-40 years.

\section{Abnormal cord findings in MRI in spinal trauma}

Cord abnormalities were present in $73 \%$ of patients. This was in near concordance with Umesh $\mathrm{C}$ Parashari et $\mathrm{al}^{2}$ which showed spinal cord abnormalities in more than $80 \%$.

Comparison of MRI findings with clinical profile of the patients

In patients with focus of cord haemorrhage all the 16 patients were graded initially as AIS A and out of them only 3 patients improved to AIS C. 13 patients showed no improvement. It may suggest that the patients with cord haemorrhage come with complete paralysis and also initial high-grade AIS did not show significant improvement.

In 15 patients with focus of cord oedema for less than $3 \mathrm{~cm}, 1$ patient was initially graded as AIS A, 2 patient were initially graded as AIS B, 1 patient were graded as AIS C and 11 patients were initially graded as AIS D. 1 patient from AIS C \& 1 patient from AIS D improved to AIS E. Other patients didn't show any significant improvement.

In 18 patients with focus of cord oedema for more than 3 $\mathrm{cm}, 13$ patients were initially graded as AIS A, 1 patient was initially graded as AIS B, 2 patients were graded as AIS C and 2 patients were initially graded as AIS D. 2 patients from AIS A \& 1 patient from AIS B improved to AIS D. Other patients didn't show any significant improvement.

Out of 100 patients, 4 patients presented with cord ischemia in which 3 patients were initially graded as AIS A \& 1 patient was initially graded as AIS B. None of the patients showed any significant improvement during discharge.

Out of 100 patients, 8 patients presented with cord transection 
in which all the 8 patients were initially graded as AIS A. None of the patients showed any significant improvement during discharge.

It may be suggested that the patients with cord ischemia $\&$ cord transection present with almost complete paralysis and also initial high-grade AIS will not show significant improvement.

In 27 patients with no cord findings, 22 patients had no clinical deficit and hence graded as AIS E (22\%). 1 patient was initially graded as AIS B. 2 patients were initially graded AIS C. 2 patients were initially graded AIS D. The patient with AIS B showed no improvement. The patient with AIS C \& AIS D showed improvement to AIS E.

This suggests that there was some amount of neurological deficits even with the cord being normal. This may be attributed to presence of non-cord abnormalities like cord compression, fractured segments, epidural hematoma, soft tissue hematoma which indirectly effect the cord like in SCIWORA (Spinal cord injury without radiographic abnormality).

\section{Correlation of MRI findings with clinical profile of patients and neurological outcome}

In our study, 12 of 100 patients (12\%) showed improvement and 88 patients $(88 \%)$ showed no improvement.

Of 41 patients with initial AIS A, only 5 cases showed improvement while 36 didn't show any improvement.

Of 16 patients with initial AIS B, only 1 case showed improvement while 15 didn't show any improvement.

Of 6 patients with initial AIS C, 3 cases showed improvement while 3 didn't show any improvement.

Of 15 patients with initial AIS D, 3 cases showed improvement while 12 didn't show any improvement.

This suggests that chances of improvement are less in patients with initial high grade AIS.

Odds ratio is 2.75 (95\% CL 0.95-36) and the Fisher's exact $P$ value is $0.0427(P<0.05)$, which is significant. It indicates that in patients with cord oedema involving $>3 \mathrm{~cm}$ of cord, chances of improvement was 2.75 times lesser than in patients with cord oedema involving $<3 \mathrm{~cm}$ of cord.

Multivariate analysis was done to see the effect of various risk factors studied on the outcome of trauma patients. It shows that over and above all the risk factors only focus of haemorrhage was significantly associated with poor prognosis (OR 6.73; 95\% CL 1.2, 38.6; $p=0.032$ ).

In a similar study, Boldin et $\mathrm{al}^{3}$ showed the effect of haemorrhage and length of hematoma on neurological impairment. They showed that patients with haemorrhage were more likely to have completed SCI at the time of follow-up (odds ratio $=2.33,95 \%$ confidence interval, 1.42 3.82). Similar to our study they also showed that presence of large haemorrhage was associated with complete SCI and showed poor prognosis.

Similar results were also shown by Andreoli C. ${ }^{4}$ They demonstrated that patients with initial ischemia, transection \& haemorrhage had poor prognosis while those with oedema had better prognosis. Flanders et $\mathrm{al}^{5}$ showed that patients without spinal cord haemorrhage had significant improvement in self-care and mobility scores compared to patients with haemorrhages. Their study revealed that rostral limit of oedema positively correlated with admission and discharge self-care scores. Poor prognostic factors were haemorrhage, long length of cord oedema and high cervical location. Selden $\mathrm{NR}^{6}$ also showed similar results - presence of long length of intra-axial hematoma and cord oedema, each associated with poor neurological outcome.

In their contrary, randomized clinical trials by Shepard $\mathrm{MJ}^{7}$ showed that MRI provides diagnostic information on degree of damage to bone and soft tissues but does not add much to diagnosis of neurological function, although they showed that presence of haemorrhage is associated with worse prognosis but did not provide any prognostic information. $\mathrm{AE}$ Flanders et $\mathrm{al}^{5}$ also showed similar result as our study. Patients with haemorrhage had lower motor scores with poor recovery $(P<0.001)$. They performed multiple regression analysis and stated that MR information on haemorrhage and oedema increases the ability to predict clinical outcome by $16.33 \%$ over that with initial clinical score alone.

Philippe Demaerel ${ }^{8}$ showed the utility of MRI in determining the management of spinal trauma. They showed that MR is also indicated for the evaluation of patients with late complications and sequelae following spinal trauma. Andreoli C. ${ }^{4}$ showed the strong correlation between MRI appearance of traumatic spinal cord injuries in acute phase and long-term recovery of motor and sensory functions. MRI is particularly useful in unconscious patients who cannot undergo motor and sensory neurological evaluation. Saifuddin $\mathrm{A}^{9}$ also established that MRI is a vital imaging technique. Selden NR showed that emergency MRI after spinal cord injury provides accurate prognostic information regarding neurological function and aids in diagnosis and treatment of cases of persistent cord compression after vertebral alignment. Slucky AV et al ${ }^{10}$ showed the correlation of MRI findings with experimental and clinical SCI with a relative predictive value to SCI pattern on MR images indicative of long-term neurological outcome.

\section{CONCLUSION}

In this study it was found that patients with cord haemorrhage, ischemia \& transection and large cord oedema had initial high grade AIS and less chance of recovery and vice versa. Focus of haemorrhage within the cord was most important prognostic factor. Patients with presence of focus of haemorrhage had more severe grade of initial AIS than those without haemorrhage with significantly more chances of retaining complete injury at follow up.

With this study it was concluded that various MRI findings in acute spinal cord injury correlate well with the neurological deficits on admission and discharge according to ASIA impairment scale

\section{REFERENCES}

1. Shih P, Fessler RG. Trauma of the Nervous System and Spinal Cord Trauma, Bradley's Neurology in Clinical Practice, 6th edition, Philadelphia, Elsevier; 2012:12121250.

2. Parashari U. C, Khanduri S, Bhadury S, Kohli N, Parihar A, Singh R, Srivastava RN, Upadhyay D. Diagnostic and Prognostic Role of MRI in Spinal 
Trauma, its Comparison and Correlation with Clinical Profile and Neurological Outcome, According to ASIA Impairment Scale. Journal of Craniovertebral Junction 2011; 2(1):17-26.

3. Boldin C, Fankhauser F, Haunschmid C, Schweighofer F, Raith J, Schwantzer G. Predicting neurologic recovery in cervical spinal cord injury with postoperative MR imaging. Spine (Phila Pa 1976). 2006;31(5):554-9.

4. Andreoli, C., Colaiacomo, M.C., Rojas Beccaglia, M., Di Biasi, C., Casciani, E., and Gualdi, G. MRI in the Acute Phase of MRI In Spinal Cord Injury Relationship Between MRI Findings and Neurological Outcome. Radiology. 2005; 110(3):636-645.

5. Flanders AE, et al. Acute Cervical Spine Trauma: Correlation of MR Imaging Finding with Degree of Neurologic Deficit. Radiology 1990; 177(2):25-33.

6. Selden NR, Quint DJ, Patel N, d'Arcy HS, Papadopoulos SM. Emergency Magnetic Resonance Imaging of Cervical Spinal Cord Injuries: Clinical Correlation and Prognosis. Neurosurgery 1999;44(4):785-92.

7. Shepard MJ, Bracken MB. Magnetic Resonance Imaging and Neurological Recovery in Acute Spinal Cord Injury: Observations from the National Acute Spinal Cord Injury Study. Spinal Cord 1999; 37:833-37.

8. Demaerel P. Magnetic Resonance Imaging OF Spinal Cord Trauma: A Pictorial Essay. Neuroradiology. 2006; 48(6):223-32.

9. Saifuddin A. MRI of Acute Spinal Trauma. Skeletal Radiology 2001; 30(1):237-46.

10. Slucky AV, Potter HG. Use of Magnetic Resonance Imaging in Spinal Trauma: Indications, Techniques, and Utility. Journal of American Academy of Orthopaedic Surgery 1998; 6(3):134-45.

Source of Support: Nil; Conflict of Interest: None

Submitted: 05-01-2020; Accepted: 31-01-2020; Published online: 22-03-2020 